\title{
Aspectos gerais do pescado no Amazonas(1)
}

\author{
E. M. S. Honda $\left({ }^{2}\right)$, C. M. Correa $\left({ }^{2}\right)$, F. P. Castelo $\left({ }^{3}\right)$ e E. A. Zapelini $\left(^{3}\right)$
}

\begin{abstract}
Resumo
A situação do pescado no Estado do Amazonas é analisada, sendo relacionados dados referentes à produção, comercialização, industrialização e consumo deste produto, principalmente na cidade de Nianaus, É apresentada uma lista dos peixes de produção significativa e a variação que ocorre du. rante as épocas de cheia e vazante dos rios. Ainda são discutidos: métodos empregados na pesca, condiçōes e manuseio do pescado desde a sua captura até o mercado consumidor.
\end{abstract}

\section{INTRODUÇÃO}

O Estado do Amazonas com a superfície de $1.564 .445 \mathrm{~km}^{2}$, dos quais 5.458 são de águas interiores, apresenta os mais variados aspectos que vão das várzeas às elevações do alto rio Negro, dos igarapés aos grandes rios, da vegetação rasteira à floresta exuberante.

Segundo dados do IBGE (1972) a população do Estado, em 1970, era de 960.934 habitantes, dos quais 314.197 habitavam o Município de Manaus, capital do Estado.

O sistema de drenagem apresenta-se bem definido nos planaltos cristalinos e no platô terciário, mas é extremamente complexo na planície aluvial, onde existe verdadeiro labirinto de rios, paranás, lagos, furos e igarapés. Sioli (1965) caracteriza três tipos de rios amamazônicos: "de água branca (ou barrenta). rios de água transparente, clara e, rios de água "preta" ou "marrom".

A ictiofauna do Amazonas é considerada das mais expressivas; apesar disto, a maior parte dos trabalhos sobre o assunto é de carater sistemático. Raros são os dados referentes ao tamanho dos estoques, produção to- tal do Estado e composição química das espécies.

No presente trabalho, procura-se dar uma visão geral da situação do pescado no Estado do Amazonas, focalizando os aspectos da sua produção, comercialização e industrialização.

\section{MATERIAL E MÉTodos}

Os dados aqui utilizados foram obtidos através de publicações da Fundação Instituto Brasileiro de Geografia e Estatística, Colônia de Pescadores Z-2, Divisão de Abastecimento da Prefeitura de Manaus, Comissão de Desonyolvimento Econômico do Amazonas (CODEAMA), Departamento de Inspeção de Produtos de Origem Animal (DIPOA), Plano de Assistência à Pesca Artesanal (PESCART) e observações realizadas pelos autores.

\section{PRODUÇÃo PESQUEIRA}

A nivel nacional, a produção total de peixes, incluindo marinhos e de água doce, no peroído de 1968-71, apresentou um crescimento gradual, ocorrendo um acréscimo de $20 \%$ no último ano, em relação ao ano base.

Através do Quadro I, verifica-se que em 1969 a produção de peixes marinhos decresceu de $0,6 \%$, enquanto que a de peixe de água doce, cresceu em $6 \%$. Nos anos seguintes, 1970 e 1971, ocorreu o inverso; a produção de peixes marinhos aumentou em 12\% e a de peixes de água doce, diminui de 4 e $10 \%$, respectivamente.

Neste quadriênio, a participação média percentual de peixes de água doce, foi da ordem de $22 \%$.

( 1 ) - Trabalho realizado em decorrência do Convênio INPA/SUDEPE e apresentado no IV Simposio Brasileiro de Alimentação e Nutrição em Botocatu, S. Paulo (26 a 30 de janeiro de 1975).

( 2 ) - Instituto Nacional de Pesquisas da Amazônia e bolsista do CNPq.

( 3 ) - Centro Regional de Pesquisas Pesqueiras de Manaus. 
QUADRO I

PRODUÇÃO DE PEIXE NO BRASIL

em ton.

Período $1968-71$

\begin{tabular}{l|r|r|r|r|r|r}
\hline Anos & $\begin{array}{c}\text { de água } \\
\text { doce }\end{array}$ & $\begin{array}{c}\text { índice do } \\
\text { “quantum" }\end{array}$ & de mar & $\begin{array}{c}\text { indice do } \\
\text { “quantum" }\end{array}$ & $\begin{array}{c}\text { Tota! } \\
\text { "quantum" }\end{array}$ \\
\hline 1968 & 97.678 & 100,0 & 316.851 & 100,0 & 414.529 & 100,0 \\
1969 & 103.649 & 106,1 & 314.892 & 99,4 & 418.541 & 100,9 \\
1970 & 93.548 & 95,7 & 355.774 & 112,2 & 449.322 & 108,4 \\
1971 & 87.899 & 90,0 & 411.382 & 112,8 & 499.281 & 120,4 \\
\hline
\end{tabular}

FONTE: Anuário Estatístico do 1.B.G.E. - 1972/1973.

A nível regional, a análise restringe-se à Amazônia Ocidental, constituída pelos Estados do Acre e Amazonas, Territórios de Rondônia e Roraima (Dec. Lei $n^{\circ}$ 291, de 28/02/1967). Pelos dados do Quadro II, o ano de 1970 apresentou o maior crescimento da produção, com um acréscimo de $5 \%$ em relação ao ano base, registrando um decréscimo de $10 \%$ no ano seguinte.
Nesta região, o Estado do Amazonas contribuiu no período analisado, com uma participação percentual média de $95,81 \%$, Rondônia com $2,41 \%$, Acre com $1,27 \%$, e Roraima com $0,51 \%$.

Ainda com referência ao citado período, o Estado do Amazonas participou com a cota média de $21 \%$ da produção brasileira total de peixes de água doce.

QUADRO II

PRODUÇĀO DE PEIXE EM TON. 1968/71

Amazônia Ocidental

\begin{tabular}{|c|c|c|c|c|c|c|c|c|c|c|}
\hline Anos & $\begin{array}{c}\text { Amazo- } \\
\text { nas }\end{array}$ & $\begin{array}{l}\text { fndice do } \\
\text { "quantm" }\end{array}$ & Acre & $\begin{array}{l}\text { fndice do } \\
\text { "quantum" }\end{array}$ & $\begin{array}{l}\text { Ron- } \\
\text { donia }\end{array}$ & $\begin{array}{l}\text { fndice do } \\
\text { "quantum" }\end{array}$ & $\begin{array}{c}\text { Rorai- } \\
\text { ma }\end{array}$ & $\begin{array}{l}\text { fndice do } \\
\text { "quantum" }\end{array}$ & Total & $\begin{array}{l}\text { Indice do } \\
\text { "quantum" }\end{array}$ \\
\hline 1938 & 20.515 & 102,0 & 245 & 160,0 & 495 & 100,0 & 107 & 100,0 & 21.362 & 100,0 \\
\hline 1969 & 20.854 & 101,6 & 274 & 101,8 & 443 & 89,5 & 107 & 100,0 & 21.678 & 101,5 \\
\hline 1970 & 21.593 & 105,2 & 258 & 105,3 & 490 & 98,9 & 120 & 112,1 & 22.461 & 105,1 \\
\hline 1971 & 18.301 & 89,2 & 295 & 120,4 & 603 & 121,8 & 96 & 89,7 & 19.298 & 90,3 \\
\hline
\end{tabular}

FCNTE: I.B.G.E: - Colônia de Pescadores Z-2.

Analisando a evclução da produção pesqueira do Amazonas nos sete últimos anos $(1968 / 74)$, observa-se que ocorreram oscilações. O triênio 1971/73 apresentou decréscimos, contudo este último foi o mais crítico. quando houve uma queda de cerca de $34 \%$ na produção de peixes. No ano seguinte, a produção apresentou um incremento percentual de $55 \%$, porém sobe apenas $2,7 \%$ em relação ao ano baše. A maior cota de produção corresponde à micro-região 7 , que abrange maior número e os mais populosos municípios, além de incluir a capital do Estado. (Quadro III).
PRINCIPAIS ESPÉCIES DE PEIXES CAPTURAdOS

Segundo Fowler (1954), existem aproximadamente 2.000 espécies de peixes na Amazônia. Contudo, este mesmo autor frisa que devem ser deduzidos deste número as sinonímias, repetições da mesma espécie nos diferentes rios, etc.

Nos mercados de Manaus, são consideradas de interesse econômico apenas 36 "espécies" sendo que destas somente 13 apresentam produção significativa. Os nomes vulgar e científico destes peixes são dados a seguir : 


\section{QUADRO III}

PRODUÇÃO DE PEIXE POR MICRO-REGIOES

Estado do Amazonas - 1969/74

em ton.

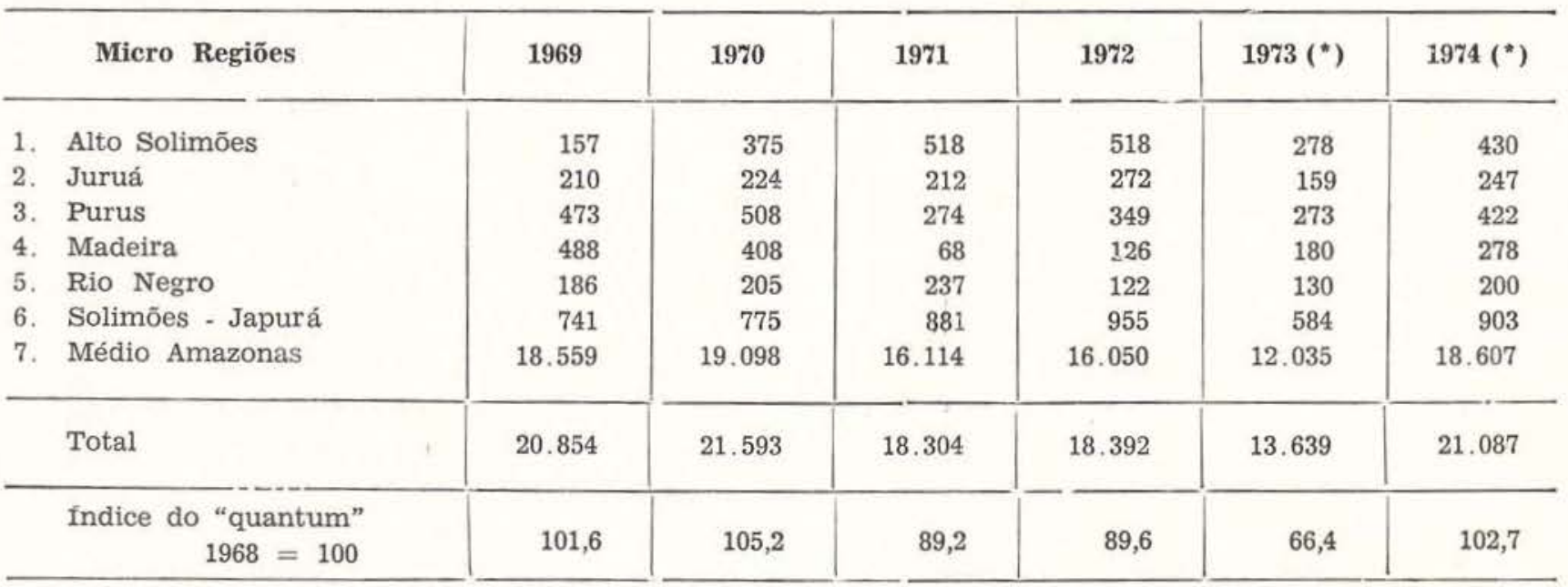

FONTE : I.B.G.E. - CODEAMA - Colônia de Pescadores Z-2.

(*) - Dados estimados.

Familia: Osteoglossidae

Pirarucu — Arapaima gigas (Cuvier)

Família : Sciaenidae

Pescada - Plagioscion squamosissimus (Heckel)

Ordem : Cypriniformes

Familia: Prochilodontidae

Curimatã - Prochilodus sp

Jaraqui — Prochilodus insignis

Schomburgk

Família: Curimatidae

Branquinha - Anodus laticeps (Valenciennes)

Família: Anostomidae

Aracu - Leporinus fasciatus (Block)

Familia: Characidae

Tambaqui - Colossoma bidens

(Spix)

Pirapitinga - Colossoma nigripinnis

(Cope)

Matrinchã - Brycon hilarii (Valenciennes)

- Sardinha - Triportheus angulatus

(Spix)

Pacu - Mietynnis sp

Ordem : Perciformes
Família : Cichlidae

$$
\begin{aligned}
& \text { Acará-açu - Astronotus occellatus } \\
& \text { (Cuvier) } \\
& \text { Tucunaré - Cichla ocellaris } \\
& \text { Schneider }
\end{aligned}
$$

A produção destas espécies no período 1970-74 é dada no Quadro IV.

Com referência à composição química de peixes da Amazônia, foram publicados dados por Gurgel (1972) e Menezes (1972). Este último autor cita que o químico $F \mathrm{M}$. Aguiar, da Diretoria de Saúde Pública do Pará, analisando quimicamente espécimes frescos de apaiari (acara-açu), pescada branca e tucuna. ré, encontrou os seguintes resultados :
Apaiari $\quad-81,72 \%$ de água; $18,60 \%$ de pro- teínas; $0,70 \%$ de gorduras e $0,69 \%$ de materiais minerais.

Pescada

branca $-82,47 \%$ de água; $15,57 \%$ de proteínas; $0,93 \%$ de gordura e $1,01 \%$ de materiais minerais.

Tucunaré $-76,22 \%$ de água; $18,45 \%$ de proteínas; $2,99 \%$ de gordura e $1,21 \%$ de materiais minerais. 


\section{QUADRO IV}

PRODUÇÃO DAS PRINCIPAIS ESPECIES CAPTURADAS E DESEMBARCADAS EM MANAUS

\begin{tabular}{|c|c|c|c|c|c|}
\hline ESPECIES & 1970 & 1971 & 1972 & 1973 & 1974 \\
\hline Tambaqui & 3.918 & 4.739 & 4.473 & 3.077 & 5.522 \\
\hline Jaraqui & 2.589 & 1.712 & 2.590 & $3.08 y$ & 3.662 \\
\hline Curimatã & 2.111 & 901 & 967 & 927 & 1.589 \\
\hline Pacu & 518 & 1.246 & 996 & 357 & 901 \\
\hline Tucunaré & 344 & 257 & 325 & 286 & 489 \\
\hline Pirapitinga & 332 & 179 & 68 & 293 & 475 \\
\hline S-rdinha & 206 & 381 & 289 & 144 & 433 \\
\hline Matrinchă & 238 & 239 & 65 & 571 & 546 \\
\hline Pirarucu & 147 & 62 & 28 & 91 & 135 \\
\hline Pescada & 75 & 107 & 177 & 101 & 133 \\
\hline Acará-açu & 26 & 42 & 32 & 45 & 100 \\
\hline Branquinha & 63 & 134 & 211 & 242 & 199 \\
\hline Aracu & - & 50 & 381 & 63 & 156 \\
\hline Peixes diversos & 292 & 1.081 & 542 & 255 & 406 \\
\hline Total & 10.859 & 11.130 & 11.144 & 9.538 & 14.746 \\
\hline
\end{tabular}

FONTE: Colônia de Pescadores Z-2.

\section{CONDIÇÕES E RECURSOS UTILIZADOS NAS ATIVIDADES PESQUEIRAS}

A maioria da frota pesqueira do Estado do Amszonas é formada por barcos, cuja capacidade varia de 1 a 10 toneladas de peixe. São embarcações que não possuem autonomia para uma viagem longa, à procura de espécies de maior valor comercial. Mesmo os barcos maiores não apresentam condiçôes adequarias, tendo os pescadores que dormir sobre as câmaras isotérmicas ou na sala de máquinas. Quase a totalidade das embarcações pesqueiras possui câmaras isotérmicas revestidas de isopor.

Estes barcos têm características bem diversas daqueles usados, para a mesma atividade, em outras regiões do Brasil. Na verdade, são apenas depósitos para armazenar, conservar e transportar o pescado. A pesca, na realidade, é efetuada em pequenas embarcações (canoas).

Segundo pesquisa sócio-econômica, realizada pelo PESCART do Amazonas junto aos pescadores e armadores de pesca, os principais métodos de captura, bem como a preferência de uso, são : rede $-72,8 \%$, anzol -
$6,3 \%$, arrastão $-5,6 \%$, tarrafa $-4,7 \%$, malhadeira $-4,0 \%$ e ainda zagaia, arpão e espinhel com menor significação.

Os processos de captura estão diretamente relacionados com a espécie a ser capturada e época do ano. Assim sendo, a rede, arrastão e malhadeira são utilizados para captura de peixes que formam cardumes, como: tambaqui, jaraqui, sardinha, curimatã, matrinchã, etc. A zagaia, para o tucunaré e acará-açu e o arpão para o pirarucu, que habitam principalmente lagos. Segundo Varoli in Menezes (1973) os processos mais utilizados durante a época de urchente são: malhadeira, espinhel e arpỏes; por ocasião da vazante é mais usada a rede.

A rede é um aparelho de pesca cujo tamathino das malhas varia pouco, contudo o diâmetro do fio é escolhido em função da espécie a ser capturada. As dimenseõs (comprimento e altura) são variáveis de acordo com o local de pesca e tamanho do cardume. A rede é formada por duas extremidades denominadas "mangas" e a parte central ("saco"), mais larga, onde se acumulam os peixes. A parte inferior possue a linha de chumbo e a da superficie, a linha das boias. As extremidades das "mangas" são presas ao calão por uma corda 
ou cabo. Do calão, saem duas cordas mais finas que se unem formando um triangulo.

A razão da preferência pelo uso da rede está baseada em :

1) grande quantidade de espécimes capturados;

2) maior facilidade de manejo;

3) as espécies de pequeno porte não sofrem grandes danos.

\section{Comercialização}

Quase toda a produção de peixe do Estado é comercializada internamente. A média do volume de peixe destinado à exportação, no período $70 / 73$ foi de 1.680 toneladas, sendo $92 \%$ de pirarucu seco salgado (segundo dados do DIPOA). A exportação de peixe congelado apresenta um percentual bastante baixo. O movimento mensal de peixe nos mercados e feiras de Manaus é expresso no Quadro V. Em 1973 , o total foi de 5.639 toneladas e em 1974 , 6.809 toneladas. Observa-se que estes totais representam $59 \%$ e $46 \%$ do total de desembarque em Manaus nesses dois anos (ver Quadro IV). Isto pode ser explicado pela existência de peixeiros ambulantes, restaurantes e peixarias que compram o produto diretamente dos barcos pesqueiros.

\section{QUADRO $V$}

MOVINENTO MENSAL DO PESCADO NOS MERCADOS E FEIRAS DE MANAUS EM TONELADAS

\begin{tabular}{|c|c|c|}
\hline MESES & 1973 & 1974 \\
\hline Janeiro & 569 & 557 \\
\hline Fevereiro & 509 & 439 \\
\hline Março & 490 & 466 \\
\hline Abril & 409 & 411 \\
\hline Maio & 320 & 467 \\
\hline Junho & 446 & 487 \\
\hline Julho & 391 & 464 \\
\hline Agosto & 452 & 520 \\
\hline Setembro & 498 & 521 \\
\hline Outubro & 520 & 518 \\
\hline Novembro & 521 & 466 \\
\hline Dezembro & 514 & 493 \\
\hline$=$ & & \\
\hline Tota 1 & 5.639 & 6.809 \\
\hline
\end{tabular}

\section{CONSUMO INTERNo "IN NATURA"}

O maior consumo no Estado é referente ao peixe "in natura", haja visto que é pequena a industrialização.

Em 1970, o consumo "per capita" de peixe para o Brasil, incluindo peixes marinhos e de água doce, foi de $4,754 \mathrm{~kg} / \mathrm{ano}$; para a Amazônia Ocidental, de $15,922 \mathrm{~kg} / \mathrm{ano}$, enquanto que para o Amazonas foi de $22,469 \mathrm{~kg} /$ ano (cerca de 4,72 vezes mais que o do Brasil).

O consumo "per capita" de peixe no Amazonas, para o período $70 / 74$, foi em média de $18,333 \mathrm{~kg} / \mathrm{ano}$, correspondendo a uma média de $50,2 \mathrm{~g} /$ dia. Em Manaus, para o mesmo período, a média do consumo "per capita" foi de $32,388 \mathrm{~kg} /$ ano ou seja $86,71 \mathrm{~g} /$ dia (Quadro VI).

\section{QUADRO VI}

CONSUMO "PER CAPITA" DE PEIXE NO ESTADO DO AMAZONAS E MANAUS

\begin{tabular}{|c|c|c|c|c|}
\hline \multirow{2}{*}{ Anos } & \multicolumn{2}{|c|}{ Amazonas } & \multicolumn{2}{|c|}{ Manaus } \\
\hline & $\mathrm{g} / \mathrm{dia}$ & g/dia & $\mathrm{kg} / \mathrm{ano}$ & $\mathrm{kg} / \mathrm{ano}$ \\
\hline 1970 & 22,469 & 61,6 & 34,582 & 94,7 \\
\hline 1971 & 18,507 & 50,7 & 33,423 & 91,5 \\
\hline 1972 & 18,084 & 49,5 & 31,569 & 86,5 \\
\hline 1973 & 13,027 & 35,7 & 25,502 & 69,9 \\
\hline 1974 & 19,579 & 53,6 & 36,865 & 101,0 \\
\hline
\end{tabular}

É necessário considerar que segundo estudo realizado pela Secretaria de Produção Rural do Estado do Amazonas (1971), há um desperdício de cerca de $30 \%$ de peixes frescos, em virtude da falta de frigoríficos para conservação. Contudo, isso não reduz de muito o indice de consumo, pois, é sabido, que no meio rural a pesca de subsistência é bastante significativa.

No Quadro VII faz-se uma análise comparativa entre os consumos de peixe e de carne bovina no Estado do Amazonas. Para estimativa do consumo de carne bovina, utilizou-se a taxa média de desfrute para o Estado (17\%) e o peso médio de carcaça $(135 \mathrm{~kg})$ relativo ao período $68 / 70$. Baseado no efetivo bovino estimou-se o consumo "per capita" de carne bovina para $70 / 72$. 
QUADRO VII

ESTADO DO AMAZONAS CONSUMO "PER CAPITA" DE CARNE BOVINA E PEIXE

\begin{tabular}{r|r|r|r|r}
\hline Anos & $\begin{array}{r}\text { Consumo } \\
\text { Peixe-Kg }\end{array}$ & $\begin{array}{r}\text { Con. carne } \\
\text { Bovina-Kg }\end{array}$ & Total & $\begin{array}{r}(\%) \\
\text { Peixe }\end{array}$ \\
\hline 1970 & 22,469 & 8,007 & 30,476 & 73,72 \\
1971 & 18,507 & 6,688 & 25,195 & 73,45 \\
1972 & 18,084 & 7,831 & 25,915 & 69,78 \\
\hline
\end{tabular}

FONTE : I.B.G.E. — CODEAMA.

O consumo médio de carne bovina "per capita" foi de cerca de $7,509 \mathrm{~kg} / \mathrm{ano}$, enquanto que o de peixe correspondeu a cerca de $19,687 \mathrm{~kg} /$ ano, representando em média $72 \%$ o consumo de peixe em relação ao consumo de carne.

\section{INFRA-ESTRUTURA DA DISTRIBUIÇÃO}

A infra-estrutura de distribuição do pescado é deveras precária. Os barcos pesqueiros realizam o desembarque do produto, à noite na praia em frente ao principal Mercado Municipal de Manaus. Este desembarque é arbitrário e não são observados quaisquer princípios de higiêne.

Os peixes são levados dos barcos até a praia em canoas; muitas vezes, lavados com água poluída no próprio local e colocados em caixas de madeira, com gelo. Em seguida são carreados aos mercados e outros locais de comercialização .

Nos bairros mais afastados o pescado é vendido por peixeiros ambulantes, em pequenos tabuleiros. Atualmente, estão sendo implantados postos de venda nos supermercados e as peixarias.

Os peixes de pequeno e médio porte, são vendidos em "cambadas" (três a seis exemplares, unidos por uma fibra vegetal através do opérculo). Outros, como o tambaqui e a pi. rapitinga são vendidos inteiros, em "bandas" ou em quartos. Somente o tucunaré, o acará-açu e o pirarucu são vendidos por quilo. A espécie mais consumida pela população de baixo poder aquisitivo, em Manaus, é o jar?qui.

A oscilação de preço do pescado é muito grande. Em 1974, na época de vazante, o cento do jaraqui foi vendido para o atacadista pelo preço de $\mathrm{Cr} \$ 5,00$ (cinco cruzeiros). Este mesmo peixe chegou ao consumidor pelo preço de Cr\$ 5,00 (cinco cruzeiros) a "cambada" com cinco exemplares. Na época de cheia, o cento deste mesmo peixe foi vendido ao atacadista por CrS 160,00 (cento e sessenta cruzeiros) e ao consumidor por $\mathrm{CrS} 8,00$ (oito cruzeiros) a "cambada" de três exemplares.

No Quadro VIII pode ser evidenciado que a maior produção ocorre nos meses de vazante, isto é, de julho a dezembro.

\section{QUADRO VIII}

PRODUCÃO MENSAL DE PEIXES DESEMBARCADOS EM MANAUS PERIODO 1970/74 EM TONELADAS

\begin{tabular}{|c|c|c|c|c|c|}
\hline Meses & 1970 & 1971 & 1972 & 1973 & 1974 \\
\hline Janeiro & 574 & 576 & 1.402 & 1.103 & 819 \\
\hline Fevereiro & 472 & 330 & 715 & 689 & 807 \\
\hline Março & 361 & 1.115 & 604 & 609 & 590 \\
\hline Abril & 678 & 381 & 481 & 673 & 662 \\
\hline Maio & 825 & 502 & 657 & 854 & 1.017 \\
\hline Junho & 514 & 536 & 646 & 852 & 1.044 \\
\hline Julho & 731 & 1.692 & 633 & 635 & 976 \\
\hline Agosto & 1.577 & 615 & 1.082 & 925 & 1. 275 \\
\hline Setembro & 1.408 & 1.483 & 1.079 & 878 & 1.350 \\
\hline Outubro & 1.447 & 1.425 & 1.336 & 776 & 1.527 \\
\hline Novembro & 1.279 & 1.223 & 1.199 & 817 & 1.578 \\
\hline Dezembro = & 993 & 1.252 & 1.310 & 727 & 3.101 \\
\hline Total & 10.859 & 11.130 & 11.144 & 9.538 & 14.746 \\
\hline
\end{tabular}

FONTE: Colônia de Pescadores $Z$ - 2 . 
INDUSTRIALIZAÇÃO E EXPORTAÇÃo

O Estado do Amazonas possui somente duas indústrias que exploram o ramo de peixes congelados, sendo uma localizada em Manaus e a outra no interior do Estado.

A primeira, Alcântara \& Cia., funciona desde 1968 e iniciou as suas atividades exportando piramutaba para o exterior; hoje em dia, a totalidade da exportação é feita para o sul do país, sendo que grande parte do produto é constituída por tucunaré, tambaqui e surubim.

A linha de processamento nesta indústria obedece à seguinte ordem : o peixe é desembarcado em local próximo da indústria, sendo previamente selecionado. Em seguida é lavado, escamado e eviscerado; sofre nova lavagem, e, logo após, é colocado em saco plástico e levado à câmara frigorífica, a um temperatura de $-30^{\circ} \mathrm{C}$. A permanência nesta câmara varia com o tamanho e peso do espécime. A estocagem do produto é feita em câmaras com temperatura de $-20^{\circ} \mathrm{C}$.

A indústria localizada no Município de Itacoatiara, Frigorífico Brasília Ltda., iniciou suas operações em 1973. Em 1974, esta indústria exportou para Brasília, 149 toneladas de peixe congelado. As espécies exportadas em maior quantidade foram: piraíba, dourado, surubim e pirapitinga.

Outro tipo de indústria encontrado no Amazonas, está ligada ao pirarucu salgado-seco. Existem no Estado, sete Entrepostos Comerciais, os quais efetuam a exportação deste tipo de peixe.

O pirarucu vem do interior do Estado, aberto em "mantas", já salgado. Em Manaus. é enviado para os Entrepostos; atualmente estes entrepostos já foram transferidos de barcos e flutuantes parados às margens dos rios, para armazens em terra. Nestes, o produto é posto novamente a secar ao sol e embalado em sacos de aniagem de juta ou similar, sendo então exportados para outros Estados, principalmente Pará, Ceará e Bahia.

O Quadro IX apresenta o total de exportação de pescado industrializado no período
1970/73 e, através dele, verifica-se que a quan. tidade de pirarucu salgado-seco é bastante significativa.

\section{QUADRO IX}

EXPORTAÇĀO DE PESCADO INDUSTRIALIZADO NO PERIODO DE $1970 / 73$

\begin{tabular}{|c|c|c|c|c|c|}
\hline \multirow{2}{*}{ ANOS } & \multicolumn{2}{|c|}{$\begin{array}{l}\text { PIRARUCU } \\
\text { SALGADO - SECO }\end{array}$} & \multirow{2}{*}{$\begin{array}{c}\text { PESCADO } \\
\text { CONGE- } \\
\text { LADO } \\
\begin{array}{c}\text { Peso } \\
\text { ton.) }\end{array}\end{array}$} & \multicolumn{2}{|c|}{$T \circ T A L$} \\
\hline & $\begin{array}{l}\text { Peso } \\
\text { Iton.) }\end{array}$ & $\begin{array}{l}\text { Valor } \mathrm{cm} \\
\mathrm{Cr} \$ 1.000\end{array}$ & & ton.) & $\begin{array}{l}\text { Valor em } \\
\text { Crs } 1.000\left({ }^{\circ}\right)\end{array}$ \\
\hline 1970 & 2.121 & 3.909 & 118 & 2.239 & 3.909 \\
\hline 1971 & 1.208 & 3.781 & 8 & 1.216 & $378 i$ \\
\hline 1972 & 1.226 & 5.062 & 166 & 1. 392 & 5.062 \\
\hline 1973 & 1.650 & 6.175 & 224 & 1.874 & 6.175 \\
\hline
\end{tabular}

FONTE : DIPOA.

(*) - Valor referente apenas ao Pirarucu Salgado-seco.

\section{CONSIDERAÇÕES FINAIS}

Desde o início da colonizaçăo do Amazonas, o homem tem sua vida condicionada pela extensa rede hidrográfica ali existente; o rio é sua via de transporte, fonte de alimentos e renda. O maior número de lugarejos e cidades é encontrado ao longo dos rios de água branca, onde a produção de peixes é bastante elevada. A atividade pesqueira é de grande importância para o Estado e o pescador artezanal é responsável pelo total da produção. O indice de consumo "per capita" anual de peixe, seja em todo o Estado do Amazonas, seja apenas na capital, é altamente significativo.

Apesar de tudo, as atividades pesqueiras não têm apresentado um crescimento constante. A inexistência de um correto sistema de controle estatístico dificulta o conhecimento real da produção pesqueira no Estado. A pesca indiscriminada durante todo $o$ ano contribui para a depredação da ictiofauna. A falta de infra-estrutura adequada de captura, armazenamento e comercialização contribuem para o estrangulamento deste subsetor da economia estadual, causando perdas e utilização parcial do produto.

Há necessidade urgente de preservar os estoques pesqueiros da região, sem que isto venha a prejudicar as atividades do pescador. Neste sentido é que são dadas as sugestões que se seguem : 
1 - Intensificar as pesquisas da biologia das espécies e da tecnologia da pesca;

2 - Organizar e aprimorar os sistemas de registro e coleta de informações e dados estatísticos;

3 - Implantar um terminal pesqueiro, objetivando melhorar a infra-estrutura de desem. barque, armazenamento e comercialização do pescado;

4 - Aprimorar e introduzir técnicas de conservação e industrialização;

5 - Estimular a exportação das espécies subutilizadas;

6 - Estudar a viabilidade de implantação da piscicultura intensiva.

\section{SUMMARY}

This is an analysis $\mathrm{O}_{\mathrm{i}}$ the situation of fishing in the State of Amazonas, giving information about the production, commercialization, and the consumption of fish, principally for the city of Manaus. A list is given of the fish most frequently found in the markets and the variation in the supply which occurs between the seasons with high water level and low water level. Methods used in fishing, and conditions, and handling of fish from the time of capture until they reach the consumer market are also discussed.

\section{BIBLIOGRAFIA CITADA}

AMAZONAS

1971 - PROPESCA. Projeto da Usina Piloto de Beneficiamento de Pescado de $\mathrm{Ma}$ naus. Secretaria da Produção Rural, $143 \mathrm{pp}$.

FOWLER, $\mathrm{H}$.

1954 - Os peixes de água doce do Brasil (4.a entrega). Arq. Zool. S. Paulo, 9.

Fundação I. B. G. E.

1972 - Sipnose Estatística do Amazonas.

GURGel, J. J.S. \& Frettas, J. V.F.

1972 - Sobre a composição química de 12 espécies de peixe de valor comercial de açudes do Nordeste Brasileiro, Bol. Técn. DNOCS, 30(1), jan./jun, Fortaleza.

MENEZES, R. S. DE

1972 - Potencial da pesca e piscicultura na Amazônia. Amazônia Brasileira em foco, 7: 34-61, jan./jun.

1973 - Recursos pesqueiros da Amazônia Legal. In: Simpósio Internacional sobre Fauna silvestre e pesca fluvial e lacustre na Amazônia (texto parcial mimeo. grafado).

SIOLI, $\mathrm{H}$.

1965 - A Limnologia e sua importância em pesquisas da Amazônia. Amazoniana, 1 (1): 11-35. 